\title{
Genomes2Drugs: Identifies Target Proteins and Lead Drugs from Proteome Data
}

\author{
David Toomey ${ }^{1}$, Heinrich C. Hoppe ${ }^{2}$, Marian P. Brennan ${ }^{1}$, Kevin B. Nolan ${ }^{3}$, Anthony J. Chubb ${ }^{1 *}$
}

1 Molecular Modelling Group, Royal College of Surgeons in Ireland, Dublin, Ireland, 2 CSIR Biosciences, Pretoria, South Africa, 3 Pharmaceutical and Medicinal Chemistry, Royal College of Surgeons in Ireland (RCSI), Dublin, Ireland

\begin{abstract}
Background: Genome sequencing and bioinformatics have provided the full hypothetical proteome of many pathogenic organisms. Advances in microarray and mass spectrometry have also yielded large output datasets of possible target proteins/genes. However, the challenge remains to identify new targets for drug discovery from this wealth of information. Further analysis includes bioinformatics and/or molecular biology tools to validate the findings. This is time consuming and expensive, and could fail to yield novel drugs if protein purification and crystallography is impossible. To pre-empt this, a researcher may want to rapidly filter the output datasets for proteins that show good homology to proteins that have already been structurally characterised or proteins that are already targets for known drugs. Critically, those researchers developing novel antibiotics need to select out the proteins that show close homology to any human proteins, as future inhibitors are likely to cross-react with the host protein, causing off-target toxicity effects later in clinical trials.

Methodology/Principal Findings: To solve many of these issues, we have developed a free online resource called Genomes2Drugs which ranks sequences to identify proteins that are (i) homologous to previously crystallized proteins or (ii) targets of known drugs, but are (iii) not homologous to human proteins. When tested using the Plasmodium falciparum malarial genome the program correctly enriched the ranked list of proteins with known drug target proteins.

Conclusions/Significance: Genomes2Drugs rapidly identifies proteins that are likely to succeed in drug discovery pipelines. This free online resource helps in the identification of potential drug targets. Importantly, the program further highlights proteins that are likely to be inhibited by FDA-approved drugs. These drugs can then be rapidly moved into Phase IV clinical studies under 'change-of-application' patents.
\end{abstract}

Citation: Toomey D, Hoppe HC, Brennan MP, Nolan KB, Chubb AJ (2009) Genomes2Drugs: Identifies Target Proteins and Lead Drugs from Proteome Data. PLoS ONE 4(7): e6195. doi:10.1371/journal.pone.0006195

Editor: Arnold Schwartz, University of Cincinnati, United States of America

Received May 21, 2009; Accepted June 12, 2009; Published July 10, 2009

Copyright: (c) 2009 Toomey et al. This is an open-access article distributed under the terms of the Creative Commons Attribution License, which permits unrestricted use, distribution, and reproduction in any medium, provided the original author and source are credited.

Funding: This work was funded by the Royal College of Surgeons in Ireland SYNERGY 2008 award and the Irish Government under its Programme for Research in Third Level Institutions Centre for Synthesis and Chemical Biology. The funders had no role in study design, data collection and analysis, decision to publish, or preparation of the manuscript.

Competing Interests: The authors have declared that no competing interests exist.

* E-mail: achubb@rcsi.ie

\section{Introduction}

The modern molecular biologist is confronted with increasingly large datasets. Genome sequencing data, proteomics data and microarray data are increasingly accessible, but difficult and laborious to interpret. Considering the investment cost of target validation, one needs to rank genome-sized output data in favour of proteins that can readily be modelled using homology modelling, as these structural models can be used in virtual high throughput screening (vHTS) of large compound libraries [1-3]. Microbiologists designing antibiotics need to rank their candidate proteins for lack of similarity with any human protein, to reduce the possibility of potentially toxic off-target side effects due to cross-reactivity between inhibitors and patient host proteins. In addition, it is now possible to screen the proteome for homology to targets of known drugs, using the DrugBank dataset [4], and propose FDA-approved drugs for rapid development to Phase IV clinical trials as these compounds are all defined as safe for human consumption. Much of the necessary search functionality is already available online [4-7]. However, the assimilation of this data into a cohesive table for analysis is non-trivial for molecular biologists unskilled in programming languages or database management. By providing a convenient online interface and summary table output, we hope to make this analysis open to a wide research audience.

\section{Materials and Methods}

Genomes2Drugs was developed using open source Java Enterprise Edition in the NetBeans IDE 6.0 programming environment and deployed on Sun Application Server [8]. The Basic Local Alignment Search Tool (BLAST) program 2.2 was obtained from the USA National Center for Biotechnology Information (NCBI). The human genome protein sequences and PDB protein sequences were also obtained from NCBI. Drug target protein sequences were obtained from the University of Alberta DrugBank website [4]. Output data files are parsed using BioJava 1.6 and the data entered into an open source MySQL 5.1 database. The test genome Plasmodium falciparum 3D7 protein sequences were obtained from the European Molecular Biology 
Laboratory - European Bioinformatics Institute (EMBL-EBI) Integr8 website (493.P_falciparum, [9]).

\section{Results}

Genomes2Drugs is a freely available web-based search engine that simultaneously searches each input protein sequence against the protein sequences of the human genome, the DrugBank dataset drug targets and the PDB protein structure database [http://mmg.rcsi.ie:8080/g2d/]. The schema for information processing is shown in Figure 1. Users can input either a single FASTA formatted protein sequence [10] or multiple sequences, either in an input box or an uploaded text file. For instance, complete proteome sequences can be downloaded from the EMBL-EBI Integr8 website [9], and uploaded into Genomes2Drugs. Screen shots of the input and output screens are shown in supplementary Figure S1 online. Users need to register and submit an email address, as processing occurs in the background. User information will remain private and will not be given to any third party. The user will be emailed when the job is complete, and can then login to download the result XML file which can be imported into Microsoft Excel as a 'As an XML list', provided the user has downloaded the 'g2d.xsd' file (available online) into the same directory. The results from a few input polypeptides can be opened

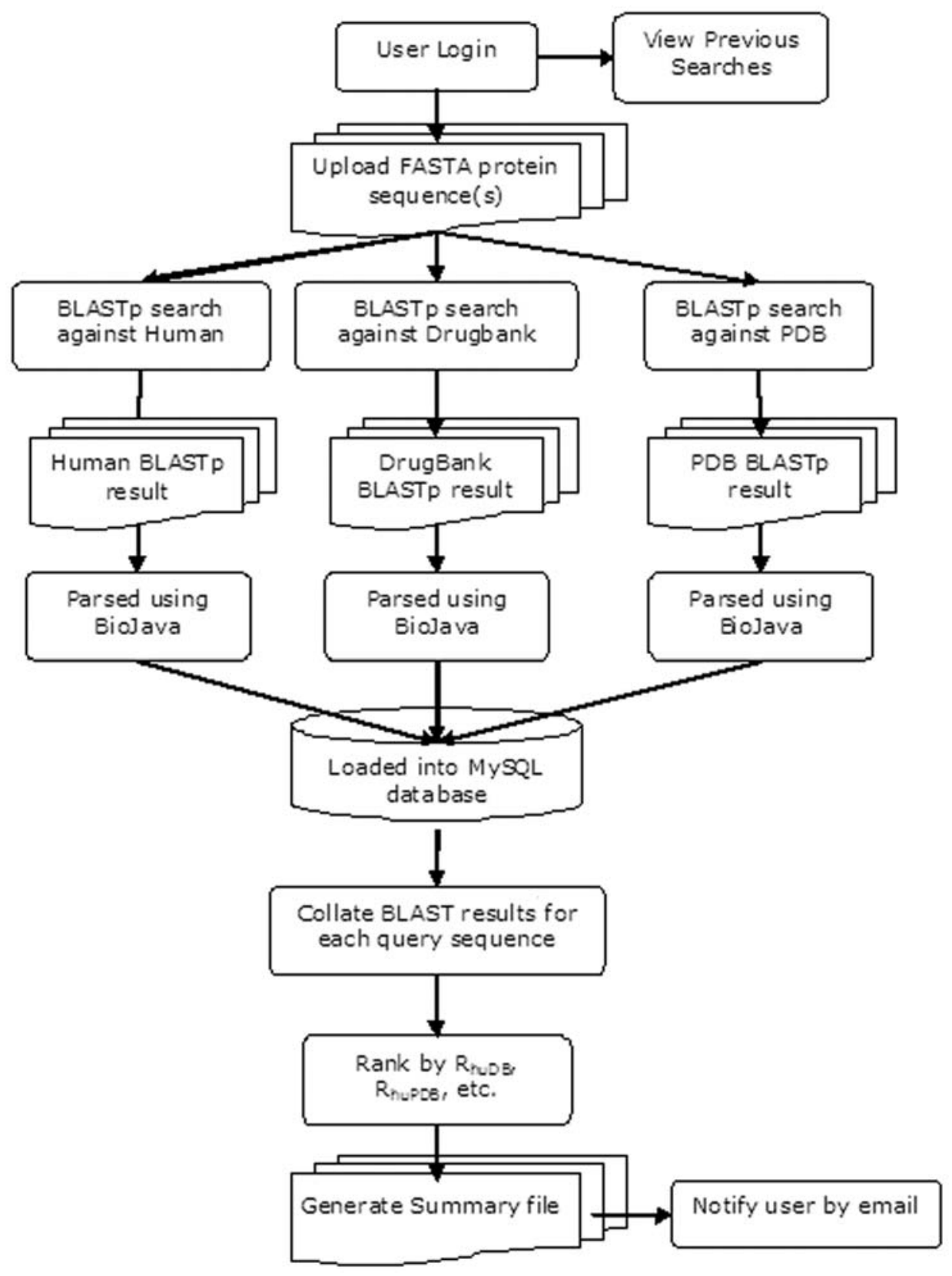

Figure 1. Schema of data processing. Genomes2Drugs is a free online resource. The web interface was written using open-source Java Enterprise Edition, BioJava 1.6 and NetBeans IDE 6.0. Input sequences are aligned against the human proteome, the PDB dataset and the DrugBank target proteins dataset. Only the best results are preserved. The resulting output files are parsed using BioJava and entered into a MySQL 5.1 database, where the results are sorted and ranked. Output XML files are generated from this data. doi:10.1371/journal.pone.0006195.g001 
in Excel, while larger genome wide searches should be opened in a database viewer like Microsoft Access, for which a viewing form is included (see supplementary Figure S1B). For easy of access to the data in Access we have included a template MDB file and XSD schema file which need to be downloaded to the same directory as the XML file. The output terms are described in Table 1. Each $\mathrm{E}_{\text {BLASTp }}$ value is derived from the optimal alignment across the genome using default settings of NCBI's freely available BLASTp algorithm [5,6]. As the best alignment score is recorded for each input protein, it follows that a poor score indicates that there is no matching protein in the comparator set. Thus a large $\mathrm{E}_{\text {BLASTp }}[-$ query vs human genome] value indicates that there is likely no match for that query protein in the human genome. Similarly, good sequence identity, with a small $\mathrm{E}_{\mathrm{BLASTp}}$ [query vs $\mathrm{PDB}$ ] value indicates that the query sequence has a close homologue in the PDB structural database. No lower limit is set for any $\mathrm{E}$ value during the alignment calculation and only the best results are shown.

The $\langle$ human expect $\rangle$ and $\langle$ PDB expect $\rangle$ columns can be used individually to rank the whole input genome for proteins showing little homology to the human genome or good homology to a protein for which the crystal structure has been determined, respectively. More conveniently, the ratio of these expect values can be used to rank the output list according to proteins that would be readily structurally modelled, while also showing little identity to any human proteins. This ratio is provided in the logarithmic (base 10) form, in the column $\mathrm{R}_{\text {huPDB }}$ (2), which has been ranked by descending value.

Table 1. Key for output file column headings.

\begin{tabular}{|c|c|}
\hline Column title & Explanation \\
\hline query_id & Unique query entry number. \\
\hline query_accession & First word of input protein title. \\
\hline query_title & Input protein title after ' $\rangle$ '. \\
\hline query_length & Number of residues in input sequence. \\
\hline RhuDB & Logarithm (base 10) of the ratio of $\langle$ human expect $\rangle$ and $\langle$ drugbank expect $\rangle$. \\
\hline RhuDBRank & Entries ranked by descending $R_{\text {huDB. }}$ \\
\hline RhuPDB & Logarithm (base 10) of the ratio of $\langle$ human expect $\rangle$ and $\langle$ PDB expect $\rangle$. \\
\hline RhuPDBRank & Entries ranked by descending $R_{\text {huPDB }}$. \\
\hline RDBPDB & Logarithm (base 10) of the ratio of $\langle$ drugbank expect $\rangle$ and $\langle$ PDB expect $\rangle$. \\
\hline RDBPDBRank & Entries ranked by descending $\mathrm{R}_{\mathrm{DBPDB}}$. \\
\hline human_accession & First word of human protein title. \\
\hline human_title & Extracted from target sequence name in BLASTp output. \\
\hline human_expect & Only optimal human/query alignment is returned, i.e. lowest BLASTp E value. \\
\hline human_rank & $\begin{array}{l}\text { Query vs Human genome alignments are ranked by descending 〈human_expect }\rangle \text {. I.e. poor/no match to the human genome is } \\
\text { scored well and given a low rank number. }\end{array}$ \\
\hline human_identities & Number of identical residues in query and human sequences. \\
\hline human_percent_identities & $(\langle\text { human identities }\rangle /\langle\text { query length }\rangle)^{*} 100$ \\
\hline human_positives & Number of homologous residues in query and human sequences. \\
\hline human_percent_positives & $(\langle\text { human positives }\rangle /\langle\text { query length }\rangle)^{*} 100$ \\
\hline pdb_accession & Protein Data Bank accession number: $\left.p d b_{1} x_{x} x_{1}\right|_{1}$ \\
\hline pdb_title & Name of protein 3-D structure. \\
\hline pdb_expect & Only optimal PDB/query alignment is returned, i.e. lowest BLASTp E value. \\
\hline pdb_rank & $\begin{array}{l}\text { Query vs Protein Data Bank sequence alignments are ranked by ascending }\langle p d b \text { expect }\rangle \text {. I.e. excellent matches with very low } \mathrm{E} \\
\text { values are scored well and given a low rank number. }\end{array}$ \\
\hline pdb_identities & Number of identical residues in query and PDB sequences. \\
\hline pdb_percent_identities & $(\langle\text { pdb_identities }\rangle /\langle\text { query length }\rangle)^{*} 100$. \\
\hline pdb_positives & Number of homologous residues in query and PDB sequences. \\
\hline pdb_percent_positives & $(\langle\text { pdb_positives }\rangle /\langle\text { query length }\rangle)^{*} 100$. \\
\hline drugbank_accession & DrugBank accession number of target protein: nnnn_all_target_protein.fasta. \\
\hline drugbank_title & Name of DrugBank target protein, including target drug accession numbers in parentheses: (DBnnnnn). \\
\hline drugbank_expect & Only optimal DrugBank/query alignment is returned, i.e. lowest BLASTp E value. \\
\hline drugbank_rank & $\begin{array}{l}\text { Query vs DrugBank sequence alignments are ranked by ascending }\left\langle p d b \_ \text {expect }\right\rangle \text {. I.e. excellent matches with very low } E \text { values are } \\
\text { scored well and given a low rank number. }\end{array}$ \\
\hline drugbank_identities & Number of identical residues in query and DrugBank sequences. \\
\hline drugbank_percent_identities & $(\langle\text { drugbank_identities }\rangle /\langle\text { query length }\rangle)^{*} 100$. \\
\hline drugbank_positives & Number of homologous residues in query and DrugBank sequences. \\
\hline drugbank_percent_positives & $(\langle\text { drugbank_positives }\rangle /\langle\text { query length }\rangle)^{*} 100$. \\
\hline
\end{tabular}


Table 2. Definition of ratio ranges and error codes.

\begin{tabular}{|c|c|c|c|}
\hline & $\mathbf{R}_{\text {huDB }}$ & $\mathbf{R}_{\text {huPDB }}$ & $\mathbf{R}_{\mathrm{DBPDB}}$ \\
\hline $\mathrm{E}_{\text {BLASTp }}\left[\right.$ [hum] $\psi$ vs. $\mathrm{E}_{\text {BLASTp }}[\mathrm{DB} / \mathrm{PDB}]^{\xi}$ & -183 to 183 & -183 to 183 & -7000 \\
\hline $\mathrm{E}_{\text {BLASTp }}[\text { hum }]^{\psi}$ vs. 'Null' DB/PDB ${ }^{\varphi}$ & -2000 & -5000 & -8000 \\
\hline 'Null' DB/PDB ${ }^{\varphi}$ vs. $E_{\text {BLASTp }}[\text { hum }]^{\psi}$ & -3000 & -6000 & -9000 \\
\hline
\end{tabular}

${ }^{*}$ BLASTp expect value of the best query/human genome alignment (null $=1000$ ).

BLASTp expect value of the best query/DrugBank alignment or query/protein data bank alignment (not null).

${ }^{\varphi}$ No alignment found between query and either DrugBank or PDB databases (null).

doi:10.1371/journal.pone.0006195.t002

The ratio values are calculated as follows:

$$
\begin{gathered}
\mathrm{R}_{\text {huDB }}=\log _{10}\left(\frac{\mathrm{E}_{\text {BLASTp }}[\text { query vs human genome }]}{\mathrm{E}_{\text {BLASTp }}[\text { query vs Drug Bank }]}\right) \\
\mathrm{R}_{\text {huPDB }}=\log _{10}\left(\frac{E_{\text {BLASTp }}[\text { query vs human genome }]}{E_{\text {BLASTp }}[\text { query vs PDB }]}\right) \\
\mathrm{R}_{\text {huDBPDB }}=\log _{10}\left(\frac{E_{\text {BLASTp }}[\text { query vs Drug Bank }]}{E_{\text {BLASTp }}[\text { query vs PDB }]}\right)
\end{gathered}
$$

Where $\mathrm{E}_{\mathrm{BLAST}}[$ is the expect value extracted from the BLASTp alignment output file using open-source BioJava [8]. The BLASTp algorithm approximates the best alignment ( $\mathrm{E}$ value $=1 \mathrm{e}-180)$ to zero. To include these data in the ratios, we set
$\mathrm{E}=0.0$ back to $\mathrm{E}=1 \mathrm{e}-180$. To include the important 'NULL' results from the human search in our ratio calculations, we arbitrarily set this to 1000 . The full range for the $\mathrm{R}_{\text {huDB }}$ and $\mathrm{R}_{\text {huPDB }}$ values is thus -183 to +183 . However, a 'NULL' result from the PDB and DrugBank database searches needs to be flagged, as these query proteins are likely to be more difficult to homology model, and do not show homology to targets of known drugs. Error messages from these ratios are defined in Table 2. The negative numbers used will rank these queries to the bottom a descending list.

Query sequences that show good homology to crystal structure template sequences, but poor/no homology to any protein within the human genome, will have high $\mathrm{R}_{\text {huPDB }}$ values. The researcher may be particularly interested in the "hypothetical" or "unknown" query proteins that are ranked well according to $\mathrm{R}_{\text {huPDB }}$ (in the top $\sim 100$ ) as these may make excellent targets for novel research into characterisation, validation, crystallography/modelling and virtual high throughput screening.

\section{P.falciparum keywords in <PDB_title>}

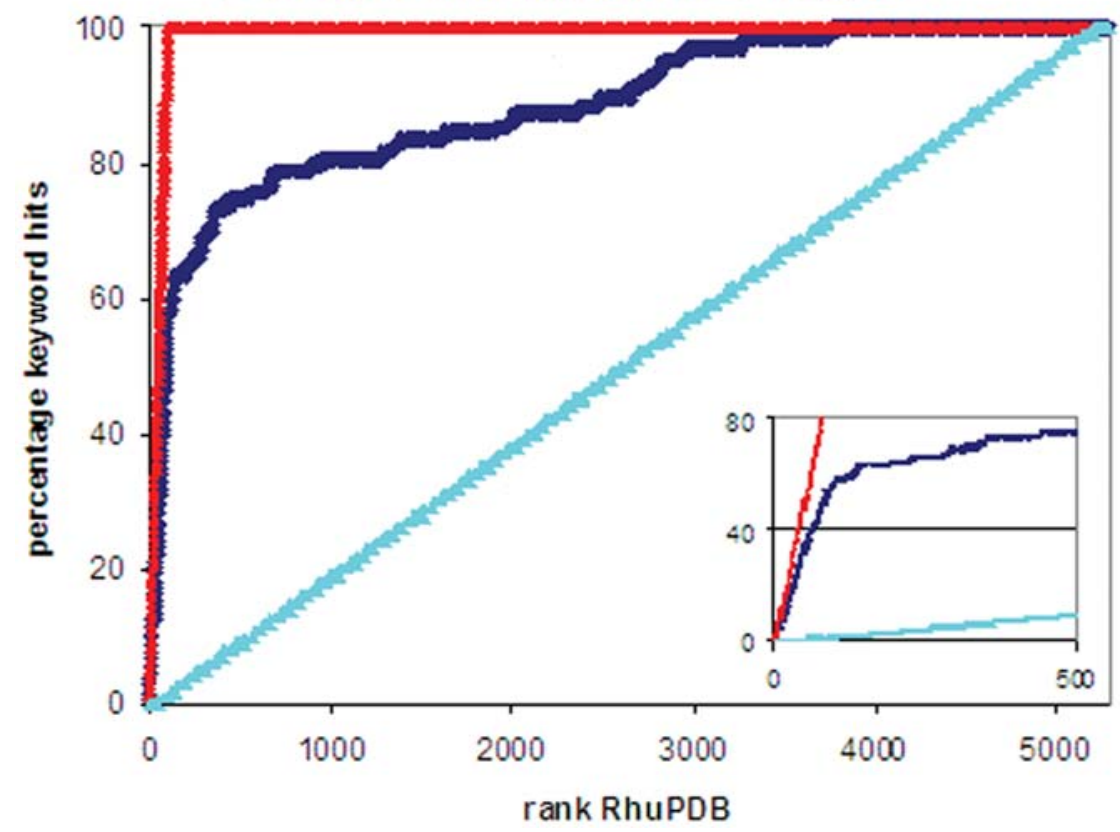

Figure 2. Enrichment of $P$. falciparum proteome by $\mathbf{R}_{\text {huPDB }}-\mathbf{P D B}$ targets. Enrichment curves plot the accumulation of user-defined 'hits' as a function of rank number. Thus in an ideal case (red line), each consecutive entry in the ascending ranked list will be a hit. Alternatively, if ranking provides no selection the hits will be distributed randomly across the genome (light blue line). The enrichment percentage as a function of rank are shown in dark blue. The 5283 proteins in the $P$. falciparum 3D7 strain test set were searched using Genomes2Drugs and ranked by $R_{\text {hupDB }} . P$. falciparum and malaria related hits from PDB were identified using keyword searching of the 〈pdb_title field, and their position in the ranked list identified. The insert, which highlights the first 500 entries, shows that almost $80 \%$ of the entries with close homology to known $P$. falciparum crystal structures were identified in the first $10 \%$ of the genome. doi:10.1371/journal.pone.0006195.g002 
A sample output from a search using the full proteome of the malaria parasite, Plasmodium falciparum, is shown in supplementary Table S1 online. The 5283 FASTA formatted protein sequences in the malarial genome were downloaded from the EMBL-EBI Interg8 website [9] and used as a test set. Of the top 50 entries as ranked by $\mathrm{R}_{\text {huPDB }}$, the majority $(68 \%)$ showed previous investigation and/or homology to crystal structures of Plasmodium falciparum proteins, indicating that this simple ranking system highlights good candidate drug targets (see Figure 2). This is further illustrated over the full genome test set in Figure 2. A query entry was defined as a 'hit' if the PDB title contained keywords associated with malaria. After ranking all 5283 test set entries according to $\mathrm{R}_{\text {huPDB }}$, the percentage of hits found is plotted as a function of rank number. Thus in the insert in Figure 2 it is clear that $\sim 80 \%$ of the hits are recovered within the first 500 entries, or $10 \%$ of the genome. The red line in Figure 2 shows an ideal case where each consecutive entry is a hit, while the light blue line shows a random distribution of hits. Interestingly, 25 of the top 50 entries are uncharacterised "hypothetical", "putative" or "unknown" proteins, which warrant further investigation as novel drug targets by virtue of the fact that they are (i) pathogen specific and (ii) similar to a structural template for homology modelling.

Similarly, query sequences homologous to known drug targets, as defined by DrugBank [4], but showing poor/no homology to any human protein, will have high $\mathrm{R}_{\text {huDB }}$ values. In Figure 3 , the full $P$. falciparum proteome test set was ranked according to $\mathrm{R}_{\mathrm{huDB}}$ and hits identified as having malaria related keywords in the best PDB match title, again indicating that high ranking entries are likely to be well characterised targets for drug discovery and development. Importantly, the same ranking showed good enrichment of known antimalarial drugs, as defined by DrugBank
(Figure 4, see listed in supplementary Table S2 online). The DrugBank hits for each query sequence are listed at the bottom of the Microsoft Access form supplied in the output of Genomes2Drugs (see supplementary Figure S1B). These compounds include experimental small molecule drugs as well as FDA (Food and Drug Administration) approved medicinal drugs, which can be purchased and tested for in vitro effectivity [4]. After ranking the $P$. falciparum test set by $\mathrm{R}_{\text {huPDB }}, 8$ of the top 50 proteins showed homology to targets of FDA approved drugs. If an FDA approved drug is found to be effective against the pathogen of interest, a 'change-of-application' patent could be sought. As all the necessary toxicology, pharmacology and dosing analysis has already been completed, Phase IV clinical trials to confirm therapeutic use may be more rapidly instigated. This could become an extremely efficient and rapid route for drug development. With a lower financial barrier to entry, this strategy could be especially important in the development of therapeutic drugs against neglected infectious diseases affecting the developing world.

\section{Discussion}

We have developed a free online resource that enriches any sized dataset of proteins of interest for those proteins likely to be most usefully in further drug discovery efforts. The program addresses the need to focus drug discovery effort on those protein targets that (i) do not show homology to proteins in the human genomes and (ii) show close homology to proteins for which the 3dimentional structure is known. As an added feature, each input protein sequence is compared to the DrugBank set of known drug targets, and may identify known drugs that are able to inhibit the protein under investigation.

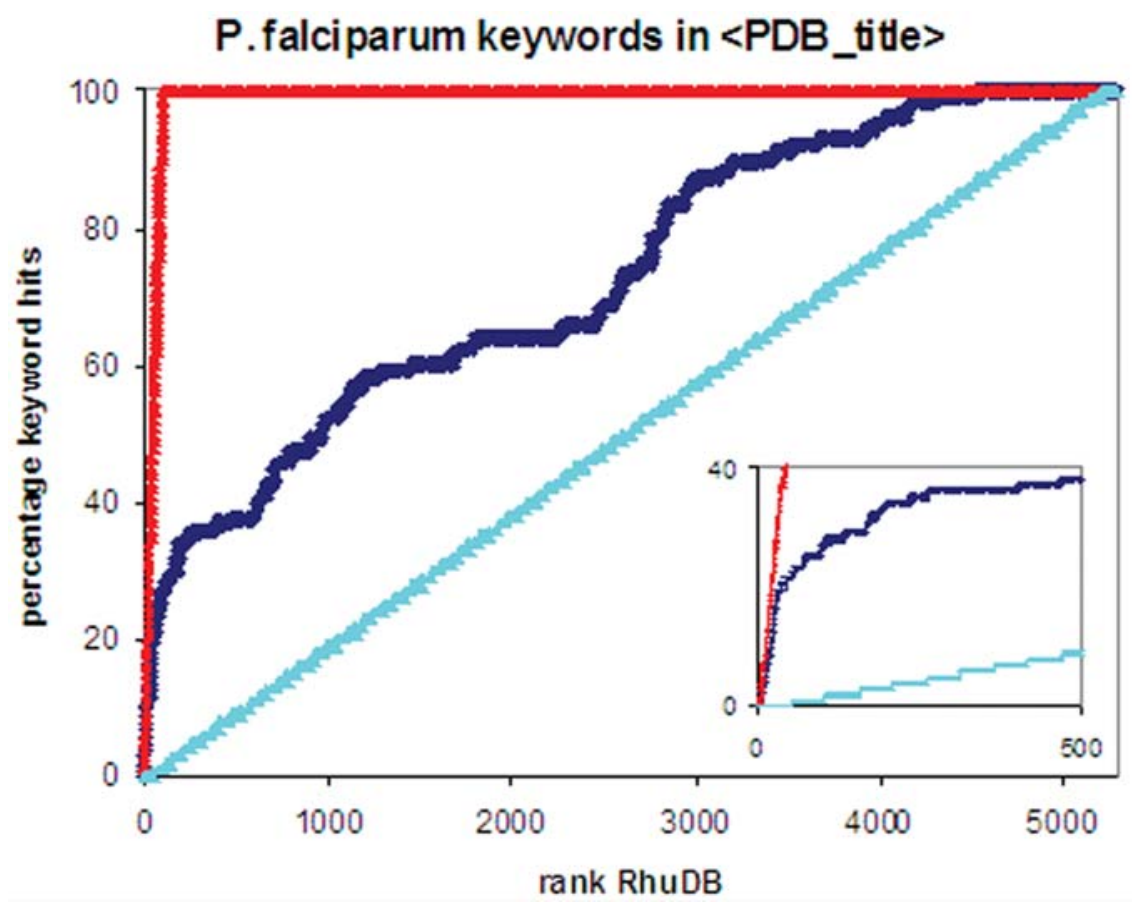

Figure 3. Enrichment of $\boldsymbol{P}$. falciparum proteome by $\mathbf{R}_{\text {huDB }}-$ PDB targets. Enrichment curves were plotted as described in Figure 2 . The 5283 protein malarial proteome was ranked by $\mathrm{R}_{\text {huDB. }}$. . falciparum and malaria related hits from PDB were identified using keyword searching of the $\left\langle p d b \_t i t l e\right\rangle$ field. The enrichment percentage as a function of rank are shown in dark blue, while the red line shows an ideal case, and the light blue line indicates a random distribution. The insert highlights the first 500 entries. doi:10.1371/journal.pone.0006195.g003 


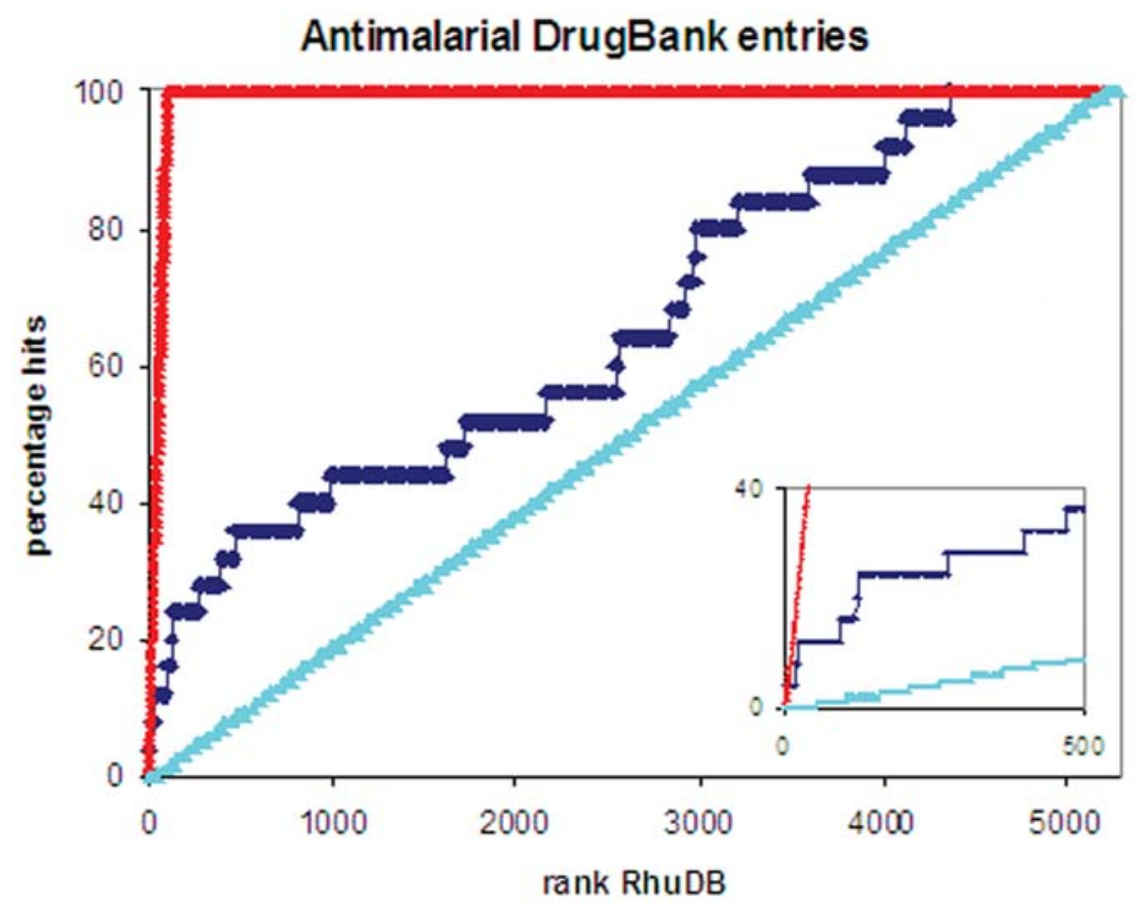

Figure 4. Enrichment of $P$. falciparum proteome by $\mathbf{R}_{\text {huDB }}$ - DrugBank targets. Enrichment curves were plotted as described in Figure 2 . The 5283 protein malarial proteome was ranked by $\mathrm{R}_{\text {huDB }}$. $P$. falciparum and malaria related hits from DrugBank were identified using keyword searching of DrugBank website [4], as shown in supplementary Table S2 online. The 〈drugbank_title〉 field entries were matched to this list of $P$. falciparum or malaria related drug targets. The enrichment percentage as a function of rank are shown in dark blue, while the red line shows an ideal case, and the light blue line indicates a random distribution. The insert highlights the first 500 entries.

doi:10.1371/journal.pone.0006195.g004

\section{Supporting Information}

Figure S1 Screen shots of the input and output of the online Genomes2Drugs tool.

Found at: doi:10.1371/journal.pone.0006195.s001 (0.58 MB PDF)

Table S1 Genomes2Drugs search of the Plasmodium falciparum proteome. The FASTA formatted proteome of the malarial parasite P. falciparum strain 3D7 was downloaded from EMBLEBI Interg8. The Genomes2Drugs output was sorted by RhuPDB. Numerous fields have been removed and abridged for clarity. Putative, uncharacterised proteins likely to be good targets for further analysis are highlighted in blue. PDB homologue titles containing the word 'plasmodium' are highlighted in yellow.

\section{References}

1. Kitchen DB, Decornez H, Furr JR, Bajorath J (2004) Docking and scoring in virtual screening for drug discovery: methods and applications. Nat Rev Drug Discov 3: 935-949.

2. Alvarez JC (2004) High-throughput docking as a source of novel drug leads. Curr Opin Chem Biol 8: 365-370.

3. Shoichet BK (2004) Virtual screening of chemical libraries. Nature 432: 862-865.

4. Wishart DS, Knox C, Guo AC, Shrivastava S, Hassanali M, et al. (2006) DrugBank: a comprehensive resource for in silico drug discovery and exploration. Nucleic Acids Res 34: D668-672.

5. Altschul SF, Madden TL, Schaffer AA, Zhang J, Zhang Z, et al. (1997) Gapped BLAST and PSI-BLAST: a new generation of protein database search programs. Nucleic Acids Res 25: 3389-3402.
DrugBank hits associated with malaria, according to NCBI PubMed, are highlighted in green.

Found at: doi:10.1371/journal.pone.0006195.s002 (0.05 MB PDF)

Table S2 DrugBank DrugCards with keywords "plasmodium" or "malaria".

Found at: doi:10.1371/journal.pone.0006195.s003 (0.01 MB PDF)

\section{Author Contributions}

Conceived and designed the experiments: DT AJC. Performed the experiments: DT. Analyzed the data: HH MPB. Contributed reagents/ materials/analysis tools: KBN. Wrote the paper: AJC. 